\title{
An elastic-plastic constitutive model for thermal shocked oxide/oxide ceramic-matrix composites
}

\author{
Zhengmao YANG ${ }^{\text {a,* }}$, Hui LIU ${ }^{\mathrm{b}}$ \\ a Institute of Mechanics, Chinese Academy of Sciences, Beijing, China \\ ${ }^{\mathrm{b}}$ School of Aerospace Engineering, Tsinghua University, Beijing, China
}

\section{A R T I C L E I N F O}

\section{Keywords:}

Ceramic-matrix composites (CMCs)

Cyclic thermal shocks

Constitutive model

Plastic potential function

Anisotropic hardening

\begin{abstract}
A B S T R A C T
The oxide/oxide ceramic-matrix composites exhibits superior mechanical properties and complex nonlinear behavior in a high-temperature environment. This paper developed a constitutive model utilising an elastoplastic modeling framework to represent the nonlinear mechanical response of the thermal shocked ox/ox-CMCs under off-axis tensile loading accurately. The model captures main features such as the effects of the thermal shock temperatures on material response. The main interest of the proposed model is the ability to predict the nonlinear mechanical response for thermal shocked ox/ox-CMCs, which is of importance for industrial applications. The validity of the constitutive model is verified by analysing the mechanical behavior of an open-hole specimen and comparing the simulation result with the experimental data.
\end{abstract}

\section{Introduction}

The oxide/oxide ceramic-matrix composites (ox/ox-CMCs) have been regarded as candidate materials in the use of gas turbine components such as flame stabilizers, combustion chamber and nozzle [1-3], due to their excellent thermomechanical properties and oxidation resistance at high temperatures compared with non-oxide CMCs. The desired service environment could be very harsh as the ox/ox-CMC components will be long-term operated at temperatures in excess of $1000{ }^{\circ} \mathrm{C}$ with varying thermomechanical loads as well as varying moisture and contaminant corrosion [4], and there are many factors affecting the mechanical properties of composite structures, such as cyclic thermal shocks [5], long-term thermal aging [6,7]. More essentially, the effects of factors on mechanical performance are normally entangled. It will be critical in determining the nonlinear stress-strain response of these composite structures when subjected to the complex service environment. In addition, CMCs possesses a relatively low fracture toughness $K_{\mathrm{IC}}$, combined with a low coefficient of thermal conductivity $k$, and can even fail under cyclic thermal shocks $[8,9]$. Consequently, considering the effects of cyclic thermal shocks, investigating the constitutive response of the ox/ox-CMCs under thermomechanical loading conditions is of great importance to aeronautical industry applications.

Numerous studies have been focused on the nonlinear mechanical behavior of the composites, and the proposed methods can be broadly categorized as (i) microscopic models and (ii) macroscopic mod-

\footnotetext{
* Corresponding author.

E-mail address: zmyang@imech.ac.cn (Z. YANG).
}

els $[10,11]$. The microscopic models take into account the microstructure of the composites [12-14], which are difficult to explicitly determine the component properties, such as matrix, fiber/matrix interface. Consequently, investigating the nonlinear behavior of the composites in the macroscopic scale especially when considering complex thermomechanical loads is more suitable [15-18]. Sun and Chen [19] introduced effective stress and plastic strain increment and developed a one-parameter flow rule for orthotropic plasticity to describe a universal nonlinear stress-strain behavior of the orthotropic composites; Vyas and Pinho [20] developed a constitutive model to represent the nonlinear mechanical response of fibre-reinforced composites by utilizing an elastoplastic modeling framework, moreover, the effect of yield stress and multi-axial loading on the applied pressure and the effect of hydrostatic pressure on the material response are taken into consideration; Zarga et al. [21] investigated the thermomechanical bending of functionally graded material (FGM) plates, and the effects of thermal load, geometrical parameters and gradient index are examined on the response of thermomechanical bending. Many other studies also investigated on the aspect of FGM [22-25]. Xie et al. [26] established a combined elastoplastic damage model to study the nonlinear mechanical behavior of 3D needled $\mathrm{C} / \mathrm{C}-\mathrm{SiC}$ composites, they proposed a new plastic potential function considering the anisotropy plastic deformation in the fiber direction for composites particularly; Liu et al. [27] developed an elastoplastic constitutive model considering variations of hardening behavior over the stress states by introducing a novel loading function considering the angle affection. The model can account for heterogeneous initial yield and complex hardening behavior but can not be used in describing the materials under complex loading such as cyclic thermal shocks. Unfortunately, all 


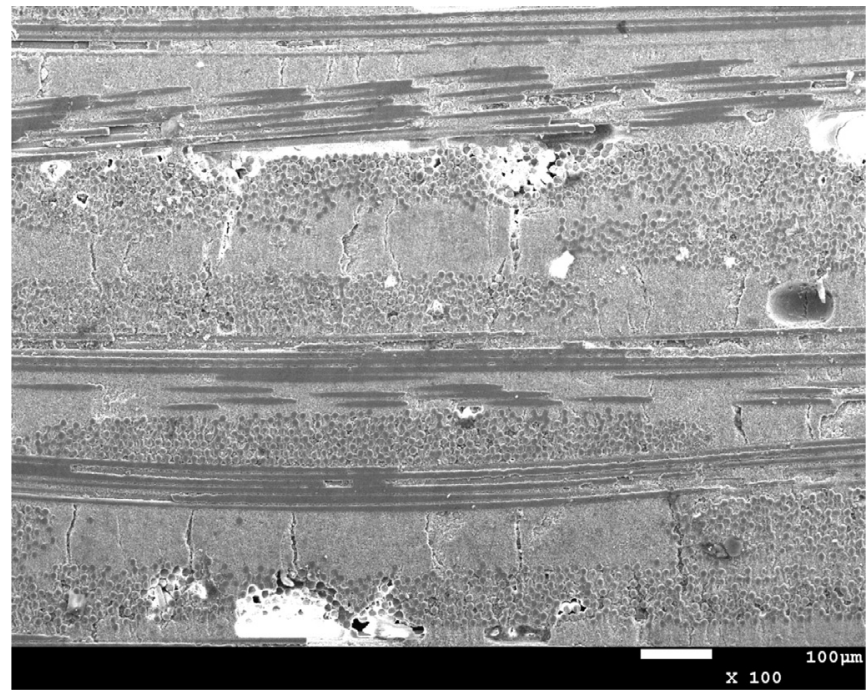

Fig. 1. SEM micrograph of a polished ox/ox-CMCs cross-section.

the works do not consider the influence of environmentally relevant variables on the material constitution, and there is no reliable model available to describe the stress-strain behaviors of the thermal shocked ox/ox-CMCs with the porous matrix, which shows the crack-deflecting behavior, similar to that in weak fiber/matrix interface composites [28-30].

The mechanical properties of cyclic thermal shocked ox/ox-CMCs have also been investigated by proceeding experimental studies. Recently, Yang et al. [31-33] investigated the thermomechanical damage in the ox/ox-CMCs under thermal shock condition and established an anisotropic damage model to describe the damage evolution and the affections to the mechanical response. However, the model was limited in the qualitative description of nonlinear mechanical behaviors and ignored the inelastic behavior in the modeling. Inelastic behavior of the thermal shocked CMCs needs much more effort in mechanical understanding and constitutive modeling.

The present work aims to develop a quantitative method to represent the mechanical behavior of the cyclic thermal shocked ox/ox-CMCs. An elastic-plastic constitutive model is introduced considering anisotropic hardening behaviors and cyclic thermal shock effects in the cyclic thermal shocked ox/ox-CMCs. An orientation-temperature-dependent hardening rule is adopted to describe the inelastic behavior of the thermal shocked ox/ox-CMCs. Different off-axis tension tests, as well as centerholed tensile panel tests, confirm reasonable accuracy of the model. The expectation is that the developed model will broader applications in the engineering design community.

\section{Materials and experiments}

\subsection{Materials}

The material studied in the present work comprises uncoated Nextel $^{\mathrm{TM}} 610$ fibers in the 8-harness satin weave, and a matrix $(85 \mathrm{wt} \%$ $\mathrm{Al}_{2} \mathrm{O}_{3}$ and $15 \mathrm{wt} \% 3 \mathrm{YSZ}$ ) made by slurry infiltration and a second sintering process for several hours at the temperature of $1000-1300{ }^{\circ} \mathrm{C}$. Some remain pores and extensive micro-cracks in the matrix were observed in scanning electron microscope (SEM) micrographs (Fig. 1). The sintering shrinkage in the matrix causes matrix tensile stress, leading the microcracks in the matrix to accommodate the stress. The sintering shrinkage cracks have been investigated in many studies [29,34], which is general in oxide/oxide composites. These cracks are normal to the fiber direction predominantly, and clustered in the matrix-rich regions.

\subsection{Thermal shock tests}

Thermal shock tests are carried out by heating the specimens to a pre-determined temperature, then holding for $10 \mathrm{~min}$ to allow for temperature equilibrium, and then drop the heated specimen into distilled water with room temperature. The maximum test temperature is set to $1100{ }^{\circ} \mathrm{C}$, as the material application is limited to $1200{ }^{\circ} \mathrm{C}$. The details of the thermal shock test which may influence the quantitative description of thermal shock effects were discussed in the authors' previous work [35]. When the ox/ox-CMCs undergo a cumulative 8-cycle thermal shock $\left(T_{\text {shock }}=1100{ }^{\circ} \mathrm{C}\right)$, the thermomechanical damage caused by the cyclic thermal shocks is nearly saturated [36]. Therefore, the 8 times cyclic thermal shocked ox/ox-CMCs have been selected for mechanical tests in this work.

\subsection{Mechanical tests}

To establish the constitutive description of the cyclic thermal shocked ox/ox-CMCs, the stress-strain relationship has to be determined from tension tests, which define the relation between the effective stress and equivalent plastic strain.

After thermal shocks, the specimens with off-axis angle $0^{\circ}, 45^{\circ}, 90^{\circ}$ were tested under monotonic tensile loading condition to recognize the stress-strain relationship. As shown in Fig. 2, the specimens are cut into a shape of $160 \mathrm{~mm} \times 20 \mathrm{~mm}$ by water jet. Two strain gages were implemented to measure the strains along $x$ axis and $y$ axis. All experiments are conducted on a MTS 809 servo-hydraulic test machine under displacement control at room temperature. The loading speed of $10^{-4} \mathrm{~mm} / \mathrm{s}$ ensures a quasi-static loading process.

On completion of the tensile tests, the micro-structures of the specimens were characterized principally using SEM, to examine the deformation in the thermal shocked composites and to study the failure mechanism.
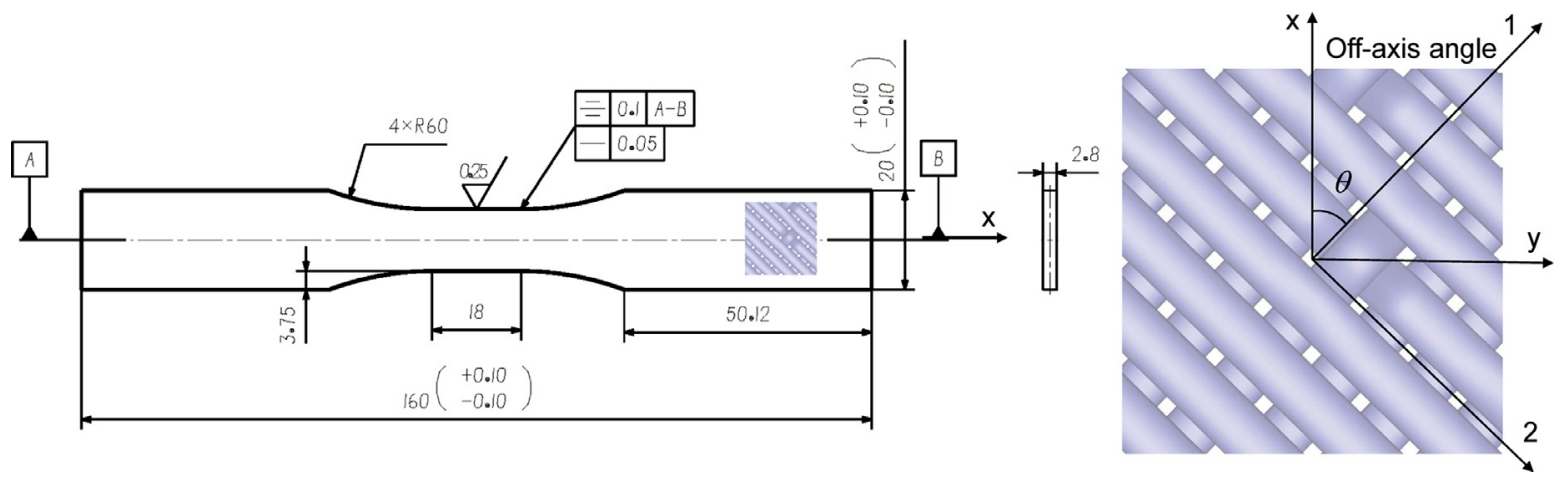

Fig. 2. The tensile specimen geometry. 
Table 1

Mechanical properties of the thermal-shocked ox/ox-CMCs.

\begin{tabular}{lllll}
\hline & Elastic modulus (GPa) & Yield stress (MPa) & Failure stress (MPa) & Failure strain (\%) \\
\hline $0^{\circ}-800{ }^{\circ} \mathrm{C}$ & 87.71 & 104.25 & 124.80 & 0.135 \\
$0^{\circ}-1000^{\circ} \mathrm{C}$ & 84.20 & 90.22 & 116.08 & 0.150 \\
$0^{\circ}-1100^{\circ} \mathrm{C}$ & 82.00 & 85.01 & 110.11 & 0.191 \\
$90^{\circ}-1100^{\circ} \mathrm{C}$ & 60.80 & 52.14 & 107.14 & 0.146 \\
$45^{\circ}-1100{ }^{\circ} \mathrm{C}$ & 40.83 & 45.18 & 48.04 & 0.16 \\
\hline
\end{tabular}

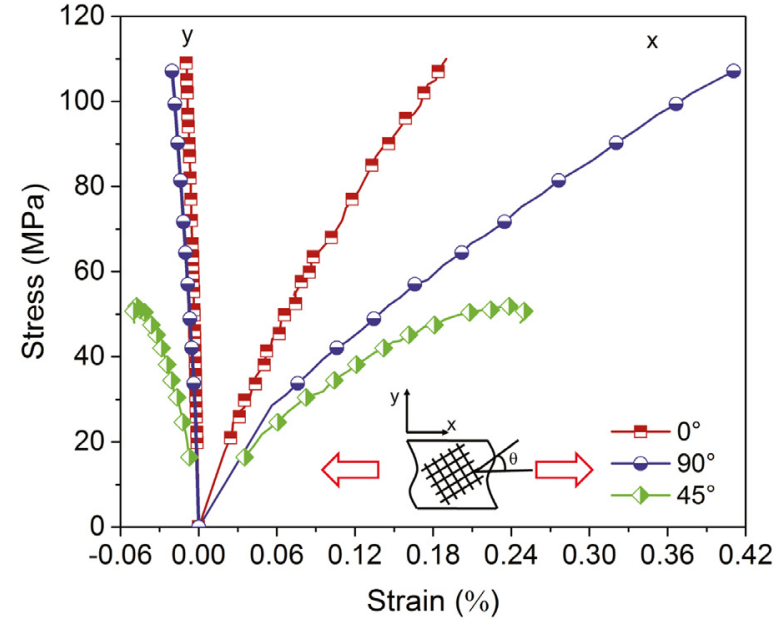

Fig. 3. The stress-strain curves of the thermal-shocked ox/ox-CMCs with different off-axis angles.

\section{Characterization of the materials}

\subsection{Mechanical properties}

Based on the experimental tests, the stress-strain curves for different off-axis angles and thermal shock temperatures of the thermal shocked ox/ox-CMCs were obtained. The characteristic material properties are, furthermore, summarized in Table 1. Obviously, the values varying with loading angles are not characteristic for the materials and have to be correlated with a material representation.

From the stress-strain response of different off-axis angle specimens shown in Fig. 3, the stress-strain curves could be divided into two regions before it fails. When the loading condition is within the first region, the stress-strain response of the materials is nearly linear and elastic, the irreversible deformation is considered to be zero. When the stress reaches to yield stress of the materials in the loading direction, the stressstrain response will come into the second stage. As the materials are weak matrix composites, crack initiates and propagates from the matrix first and permanent deformation will begin to accumulate. The fibre plies in the $90^{\circ}$ direction are much more inflected than in $0^{\circ}$ direction because of the weaving technique, leading to reduction of the effective fibre volume fraction in $90^{\circ}$ direction, and the elastic modulus in the $90^{\circ}$ direction is small than that in the $0^{\circ}$ direction.

Fig. 4 shows the stress-strain response of the thermal shocked ox/oxCMCs with different thermal shock temperatures. As shock temperatures increase, the elastic modulus decreases, due to the micro-cracks and delamination caused by thermal shocks, and the yield stress and the failure stress also decrease.

\subsection{The failure mechanism}

The failure mechanism of the thermal shocked ox/ox-CMCs is related to the loading conditions. For $0^{\circ}$ monotonic tensile specimen, the fracture surface is flat and perpendicular to loading direction yet for

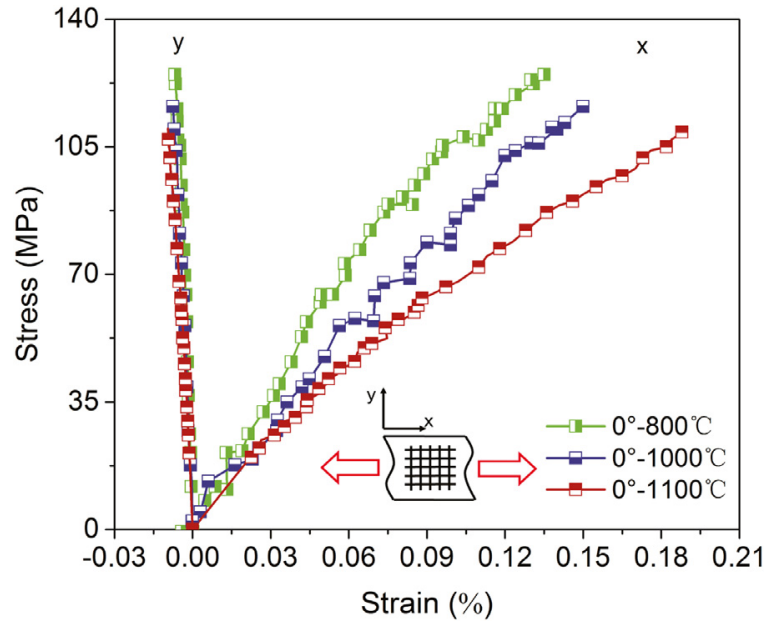

Fig. 4. The stress-strain curves of the thermal-shocked ox/ox-CMCs with different thermal shock temperatures.

$45^{\circ}$ monotonic tensile specimen the fracture surface is irregular and not perpendicular to the loading direction. Furthermore, more information would be obtained by SEM observations of different fracture surfaces.

Fig. 5 shows the fracture surface of the $0^{\circ}$ on-axis specimen, which is relatively smooth and the main feature of the fracture surface is cracks in matrix. Cracks initiate in matrix because the strength of matrix is much lower than fibers. When cracks approach fibers, they will defect at the interface between fibers and matrix and then extend along the interface because of the high strength of fibers. Some longitudinal fibers will be pulled out during the loading process when the matrix surrounded these fibers is broken enough and fibers lose supporting of matrix. When the density of cracks in matrix achieves a critical value, the material loses bearing capacity and fails in the macro-scale immediately.

The fracture surface of $45^{\circ}$ specimen is much different from that described above, as illustrated in Fig. 5(b). Because of the in-plane shear stress along with the interface between matrix and fiber, induced by the relative sliding between the interfaces, the phenomenon of fiber bundle pull-outs and single fiber pull-out can be observed. The shear stress along the interface between fiber and matrix has a great influence on the failure modes of the off-axis tension specimens.

\section{Constitutive model}

To describe the macroscopic nonlinear behavior of the elementary orthotropic thermal shocked ox/ox-CMCs under tensile loading, an elastic-plastic constitutive model was proposed in this section, which includes a plastic part for describing the plastic behavior under tensile loading.

\subsection{Plasticity framework}

In the framework of elastic-plastic mechanics, the total strain increment can be divided into two parts, the elastic part and the plastic part:

$\mathrm{d} \varepsilon=\mathrm{d} \varepsilon_{\mathrm{e}}+\mathrm{d} \varepsilon_{\mathrm{p}}$. 

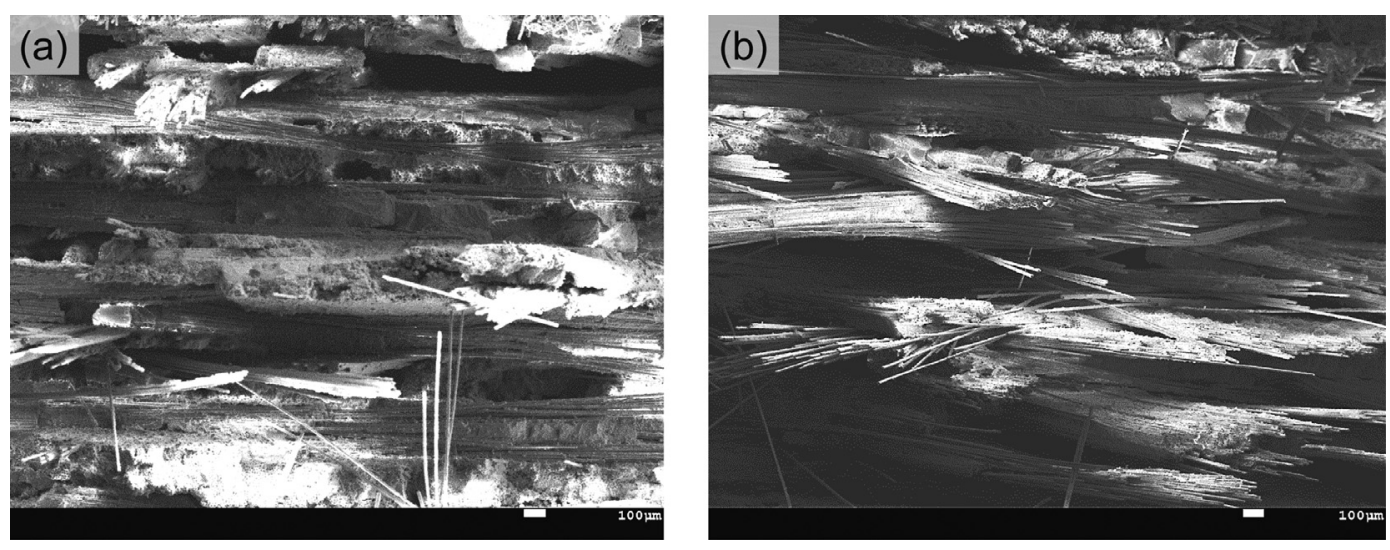

Fig. 5. The SEM of fracture surfaces for the thermal-shocked ox/ox-CMCs: (a) $0^{\circ}$ on-axis specimen; (b) $45^{\circ}$ off-axis specimen.

The elastic part $\mathrm{d} \varepsilon_{e}$ is calculated based on elasticity theory of composites and the plastic part $\mathrm{d} \varepsilon_{p}$ is discussed within the framework of plasticity.

Generally, when the effective stress reaches the yield stress, the material strain hardens. It is called hardening because the stress is increasing relative to perfect plastic behaviour, then the yield function is defined by,

$F(\sigma, p)=f\left(\sigma_{i j}\right)-\sigma_{\mathrm{y}}$

where $f\left(\sigma_{i j}\right)$ is the plastic potential function expressed the effective stress, $\sigma_{y}$ is the yield stress. The yield criterion is given by $F(\sigma, p)=0$ for plastic deformation. when considering hardening, the yield stress can increase, as a function of effective plastic strain, $\bar{\varepsilon}_{\mathrm{p}}$. It is therefore convenient to write the plastic yield function as

$F(\sigma, p)=f\left(\sigma_{i j}\right)-\kappa\left(\bar{\varepsilon}_{p}\right)=0$

here $\kappa\left(\bar{\varepsilon}_{p}\right)$ is the hardening law to describe the evolution of yield stress. The potential function is generalized to the woven composites with two principal directions, as

$f\left(\sigma_{i j}\right)=\sqrt{a_{1} \sigma_{11}^{2}+a_{2} \sigma_{22}^{2}+\sigma_{12}^{2}}=\bar{\sigma}$

with two model parameters, $a_{1}, a_{2}$ related to the anisotropy of the composites and need to be determined by experimental data. The stress components above are defined in the material principal coordinate system, and $\bar{\sigma}$ is the effective stress.

It is assumed that only if the plastic potential function is larger than or equal zero, the stress state reaches the yield surface, the plastic strain will appear during the loading process. Based on the associated plastic flow rule, the plastic strain can be obtained,

$\mathrm{d} \varepsilon_{i j}^{\mathrm{p}}=\mathrm{d} \lambda \frac{\partial f}{\partial \sigma_{i j}}$

in which $\mathrm{d} \lambda$ is the scalar proportionality coefficient which determines the increment of the plastic flow. Then, the plastic strains become:

$\mathrm{d} \varepsilon_{11}^{\mathrm{p}}=\frac{\partial f}{\partial \sigma_{11}} \mathrm{~d} \lambda=\frac{a_{1} \sigma_{11}}{\bar{\sigma}} \mathrm{d} \lambda$

$\mathrm{d} \varepsilon_{22}^{\mathrm{p}}=\frac{\partial f}{\partial \sigma_{22}} \mathrm{~d} \lambda=\frac{a_{2} \sigma_{22}}{\bar{\sigma}} \mathrm{d} \lambda$

$\mathrm{d} \varepsilon_{12}^{\mathrm{p}}=\frac{\partial f}{\partial \sigma_{12}} \mathrm{~d} \lambda=\frac{\sigma_{12}}{\bar{\sigma}} \mathrm{d} \lambda$

According to the plasticity theory, the plastic work increment of unit volume is expressed as,

$\mathrm{d} W_{\mathrm{p}}=\bar{\sigma} \mathrm{d} \bar{\varepsilon}_{\mathrm{p}}=\sigma_{i j} \mathrm{~d} \varepsilon_{i j}^{\mathrm{p}}$.

above $\bar{\sigma}$ is the effective stress calculated by the yield function, $\mathrm{d} \bar{\varepsilon}_{\mathrm{p}}$ is the equivalent plastic strain increment, $\sigma_{i j}$ is the stress component, $\mathrm{d} \varepsilon_{i j}^{\mathrm{p}}$ is the plastic strain tensor increment.
Submitting the flow rule into Eq. (7) follows

$\mathrm{d} \bar{\varepsilon}_{\mathrm{p}}=\mathrm{d} \lambda$.

which implies that the equivalent plastic strain increment is the proportionality factor. The power law is widely used to describe the hardening rule of the composite materials. Then the hardening function can be written as

$\kappa\left(\bar{\varepsilon}_{\mathrm{p}}\right)=R_{0}+A\left(\varepsilon_{\mathrm{p}}\right)^{n}=\bar{\sigma}$

where $R_{0}, A$ and $n$ are parameters to fit the experimental hardening curve.

\subsection{Representation of the off-axis loads}

The experimental data of the off-axis tension tests are used to represent the off-axis behavior of the thermal shocked ox/ox-CMCs. According to the stress transformation [37], the stress components in principal material directions under uniaxial loading experiments can be written as

$\sigma_{11}=\sigma_{\theta} \cos ^{2} \theta$,

$\sigma_{22}=\sigma_{\theta} \sin ^{2} \theta$,

$\sigma_{12}=-\sigma_{\theta} \cos \theta \sin \theta$.

where $\sigma_{\theta}$ is uniaxial stress in the loading direction.

Submitting the stress components in the principal material coordinate system into the expression of the effective stress $\bar{\sigma}$, then,

$\bar{\sigma}=\sigma_{\theta} h(\theta)$

where $h(\theta)$ describes effects of the loading direction for the uniaxial tension, as

$h(\theta)=\sqrt{a_{1} \cos ^{4} \theta+a_{2} \sin ^{4} \theta+\cos ^{2} \theta \sin ^{2} \theta}$.

Eq. (11) establishes the relationship between effective stress and the tensile stress in the off-axis tension test. In analogy to the derivation of the stress representations, the strain transformation can be written as

$\mathrm{d} \varepsilon_{\theta}^{\mathrm{p}}=\mathrm{d} \varepsilon_{11}^{\mathrm{p}} \cos ^{2} \theta+\mathrm{d} \varepsilon_{22}^{\mathrm{p}} \sin ^{2} \theta-\mathrm{d} \varepsilon_{12}^{\mathrm{p}} \cos \theta \sin \theta$

Substituting Eq. (11) into the equivalence of the plastic work Eq. (7), the equivalence plastic strain increment is derived:

$\mathrm{d} \bar{\varepsilon}_{\mathrm{p}}=\frac{\mathrm{d} \varepsilon_{\theta}^{\mathrm{p}}}{h(\theta)}$.

Similar to Eq. (9), $\sigma_{\theta}$ is expressed as a function of the off-axis strain $\varepsilon_{\theta}^{\mathrm{p}}$,

$\sigma_{\theta}=\kappa_{\theta}\left(\varepsilon_{\theta}^{\mathrm{p}}\right)$ 


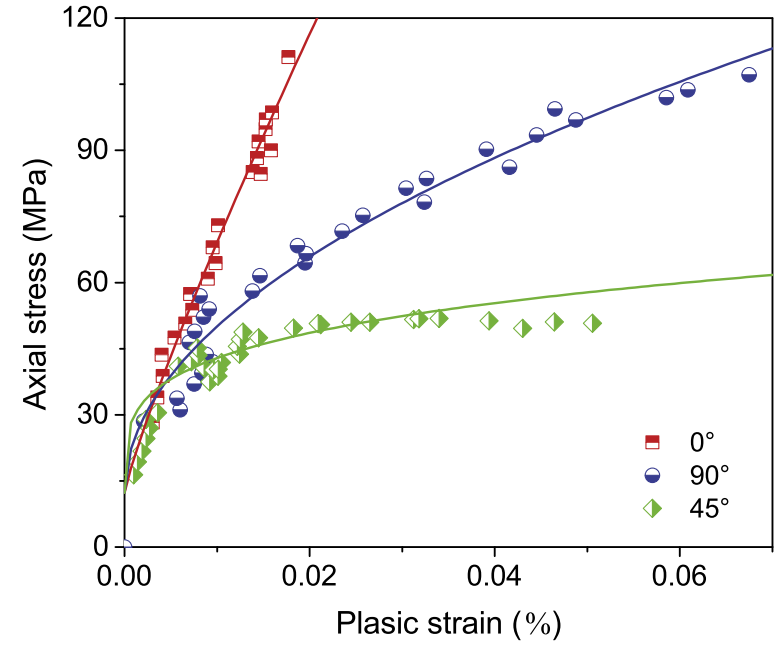

Fig. 6. The axial stress-plastic strain curves of different off-axis angles.

Table 2

Fitting parameters of different loading conditions.

\begin{tabular}{llll}
\hline Off-axis angle & $A_{\theta}$ & $n_{\theta}$ & $R_{\theta}^{0}$ \\
\hline $0^{\circ}$ & 177,600 & 0.87 & 12.33 \\
$90^{\circ}$ & 3956 & 0.51 & 12.33 \\
$45^{\circ}$ & 297.1 & 0.25 & 12.33 \\
\hline
\end{tabular}

where $\kappa_{\theta}$ is the hardening function for the present off-axis angle. Combining Eqs. (9) and (14),

$\bar{\sigma}=\kappa_{\theta}\left(\varepsilon_{\theta}^{\mathrm{p}}\right) h(\theta)=\kappa_{\theta}\left(\bar{\varepsilon}_{\mathrm{p}} h(\theta)\right) h(\theta)$

Eq. (15) denotes the stress-strain relation depending on the off-axis angle formally. Due to the heterogeneity of the material behavior, the effective stress is not uniquely determined by the equivalent plastic strain but also related to the off-axis loading angle explicitly.

According to Eqs. (9) and (15), the hardening function $\kappa\left(\bar{\varepsilon}_{\mathrm{p}}\right)$ for different off-axis experiments could be expressed as,

$\kappa_{\theta}=R_{\theta}^{0}+A_{\theta}\left(\varepsilon_{\theta}^{p}\right)^{n_{\theta}}$

where $R_{\theta}^{0}, A_{\theta}$ and $n_{\theta}$ are the fitting parameters related to the off-axis angle and can be determined from experimental data. Then the hardening function could be determined by the method mentioned above based on the fitting results for $\sigma_{\theta}-\varepsilon_{\theta}^{\mathrm{p}}$ relationships of different conditions. Fig. 6 shows $\sigma_{\theta}-\varepsilon_{\theta}^{\mathrm{p}}$ curves and their fitting results for different off-axis monotonic loading tests. The identified fitting parameters are summarized in Table 2, in which $R_{\theta}^{0}=12.33$.

\subsection{Representation of the thermal shock temperatures}

According to the phenomenon mentioned above, not only the equivalent plastic strain, but the thermal shock states will have effects on the hardening behavior of the thermal shocked ox/ox-CMCs. The parameters $A$ and $n$ in the hardening function vary with the thermal shock temperatures as depicted in Fig. 7.

It should be noted that the hardening parameters $A$ and $n$ closely relate to the thermal shock conditions and this result indicated that an invariable master-curve can not describe the anisotropic hardening behaviors of the thermal shocked ox/ox-CMCs precisely. Then $A$ and $n$ are expressed as functions of the thermal shock temperature $T_{\text {shock}}$ :

$A=A_{1} \chi+A_{0}, n=n_{1} \chi+n_{0}$

where $\chi$ is the normalized thermal shock temperature, $\chi=T_{\text {shock }} / T_{\text {ref }}$, here $T_{\text {ref }}$ is set to $400{ }^{\circ} \mathrm{C}$ referring to [36]. The fitting results of $A$ and $n$ are shown in Fig. 8, which can be used to determine the hardening rule.

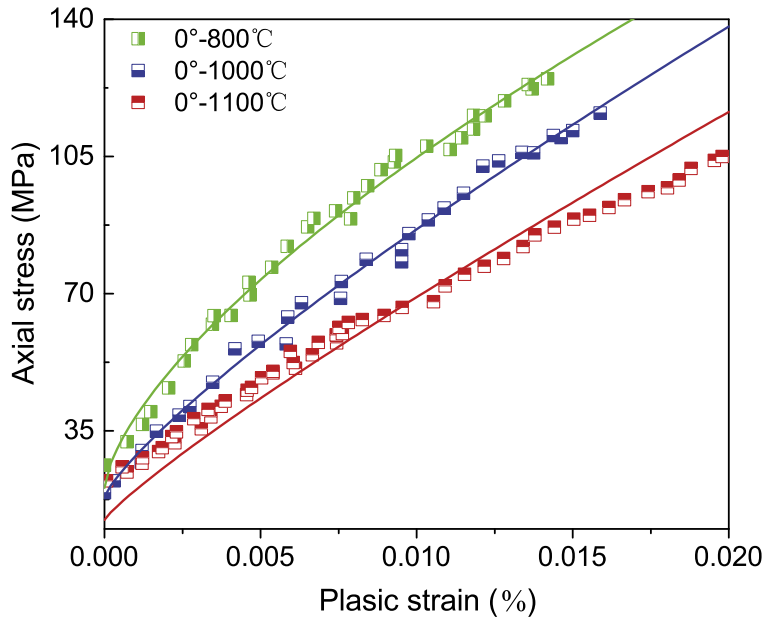

Fig. 7. The axial stress-plastic strain curves of different shock temperatures.

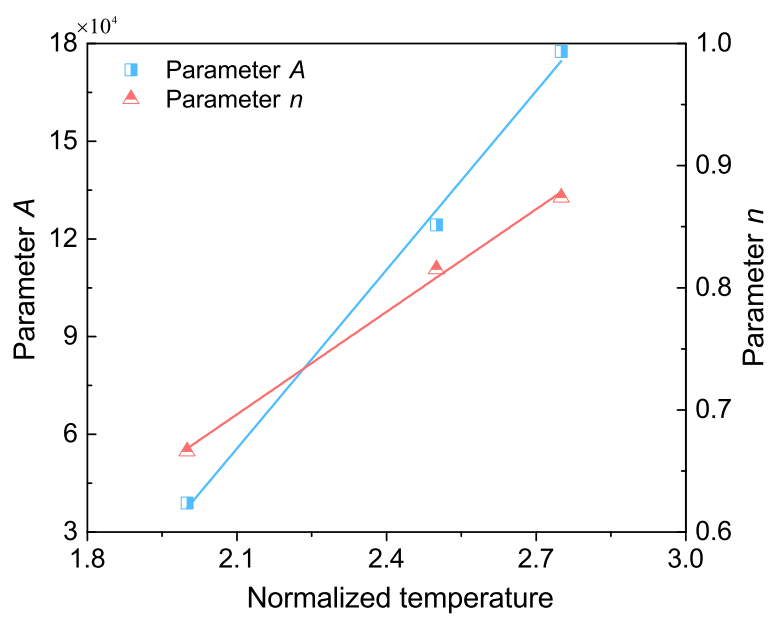

Fig. 8. The parameter distribution with the thermal shock temperatures.

Considering the $R_{0}$ as the initial stress for plastic strain, $R_{0}$ remains constant for different off-axis angles, however, as the thermal shock temperature increases, $R_{0}$ changes, implying that the effect of thermal shocks may change the initial stress for plastic strain. To characterize the relationship between $R_{0}$ and the thermal shock temperature, $R_{0}$ can be defined as a function of $\chi$ using power law, which is shown in Fig. 9.

\subsection{Results and discussion}

After determining the parameters $a_{1}, a_{2}$ in the plastic potential function using the experimental data, the yield surface of the proposed elastic-plastic constitutive model can be obtained. Fig. 11 shows the comparison of the proposed plastic yield surface and the von Mises plastic yield surface under the effective stress $\bar{\sigma}=50 \mathrm{MPa}$. The proposed yield surface plotted in red lines is surrounded by the outside of the von Mises yield surface, which indicates that the stress components of ox/ox-CMCs are higher than the general metal materials which can be represented by the von Mises yield surface when the materials reach the yield stage. Besides, it should be noted that the general plastic potential functions are inapplicable for the composite materials, especially the porous matrix materials such as the ox/ox-CMCs, which may due to the complex structures and different component material properties for the composites.

As the parameters in the hardening function have been discussed and calculated for different loading conditions, a complete elastic-plastic 


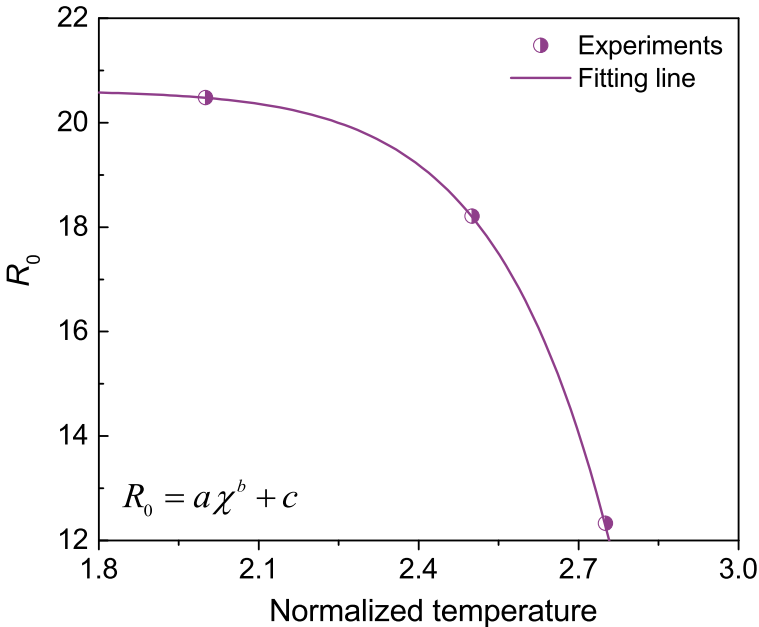

Fig. 9. The relationship between $R_{0}$ and the thermal shock temperatures.

constitutive model is obtained to describe the mechanical behaviors of the thermal shocked ox/ox-CMCs. Fig. 10 displays the predictions of the mechanical response for the thermal shocked ox/ox-CMCs, calculated by the Matlab code. The predicted stress-strain curves of uniaxial tension loadings with different off-axis angles $0^{\circ} / 45^{\circ} / 90^{\circ}$ shown in Fig. 10(a) agrees well with the experimental data at the beginning of the elastic stage, where the symbols represent the test data point and the curves represent the results of predictions. However, with the strain increasing, the predicted curves becomes slightly lower than the experimental data in the x-direction, which may due to the calculation errors for the parameter $A$ and $n$ in hardening law, as well as that the ox/ox-CMCs are the porous matrix materials, and the strength of matrix is much lower than that of the fiber, so that the matrix will fail firstly and the fiber starts to bear the most of the load when suffering tensile loading, and the modulus of fiber is high than the matrix, leading to the experimental data slightly higher than the simulation results. Besides, the predicted results are compared with the results of other model [27], which is plotted by the dot line in the diagram. The model introduced by Liu et al. is also used for the ox/ox-CMCs, and it can be indicated that Liu's model predicts well in the elastic stage, but has larger errors when predicting the plastic stage, which is due to that the model didn't consider the effects of the cyclic thermal shocks.

Moreover, the predicted results compared with the experiments are shown in Fig. 10(b) with different cyclic thermal shock temperatures.

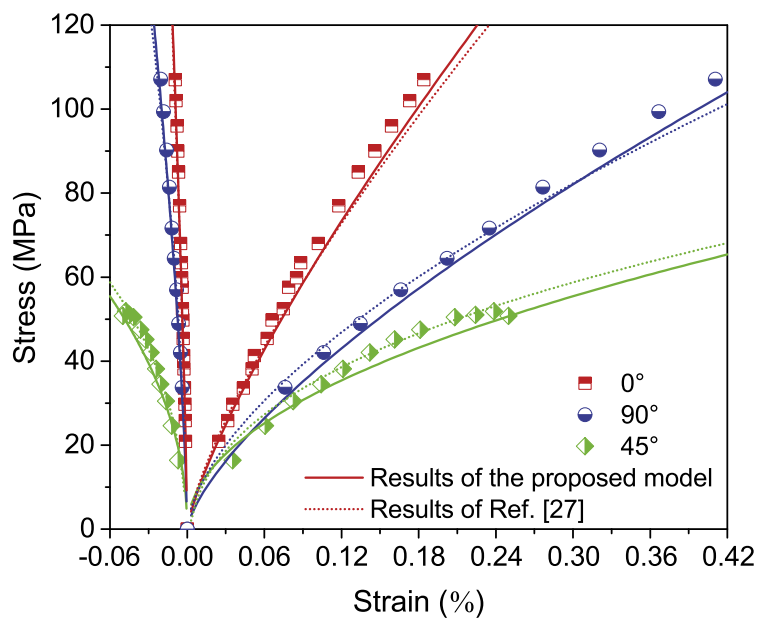

(a) Off-axis angles

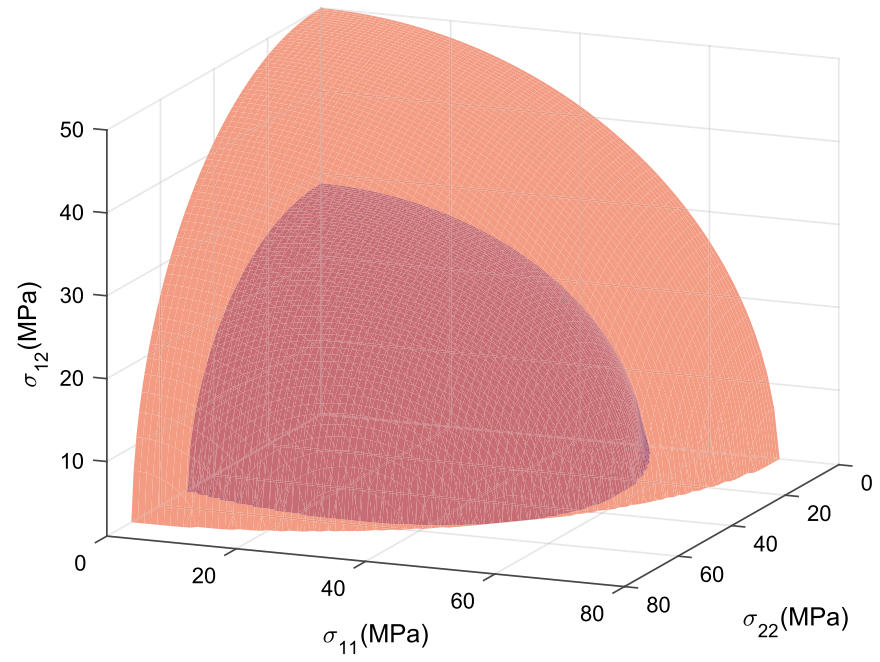

Fig. 11. Comparison of the proposed yield surface and von Mises yield surface.

The results also show a good agreement with the experimental data, and the curves in the plastic stage are also slightly lower than the experiments, which may also due to the reasons mentioned above. The stress-strain responses can be described accurately according to the comparison between experiments and calculations.

\section{Model validation}

The proposed constitutive model was coded into the user-defined material subroutine (UMAT) of the finite element software ABAQUS. By comparing the calculated results with the experimental data, the predicted outcomes determined by the proposed constitutive model are consistent with the experimental results accurately.

\subsection{Experiments}

To further verify the accuracy of the proposed model, an open-hole specimen was tested under tensile loading. The experiment was also conducted in MTS 809 under displacement control at a loading speed of $0.001 \mathrm{~mm} / \mathrm{s}$. Two positions in the specimen were selected to be the representative locations and strains in the representative locations were measured by a strain rosette. The specimen and representative locations are displayed in Fig. 12.

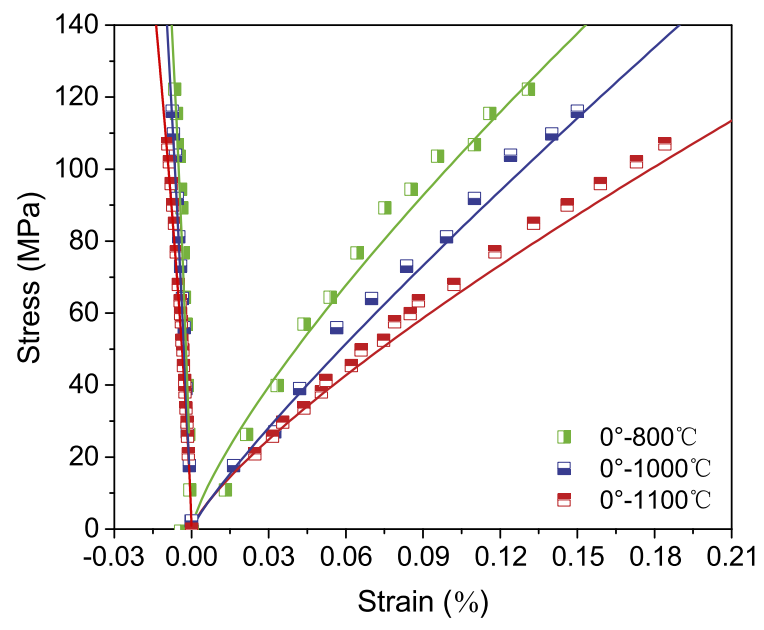

(b) Thermal shock temperatures

Fig. 10. The prediction of stress-strain response for uniaxial tension experiments. 


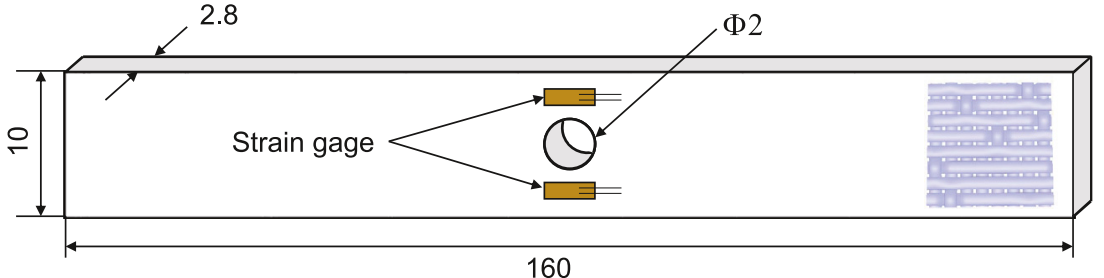

Fig. 12. Schematic of the open-hole specimen.

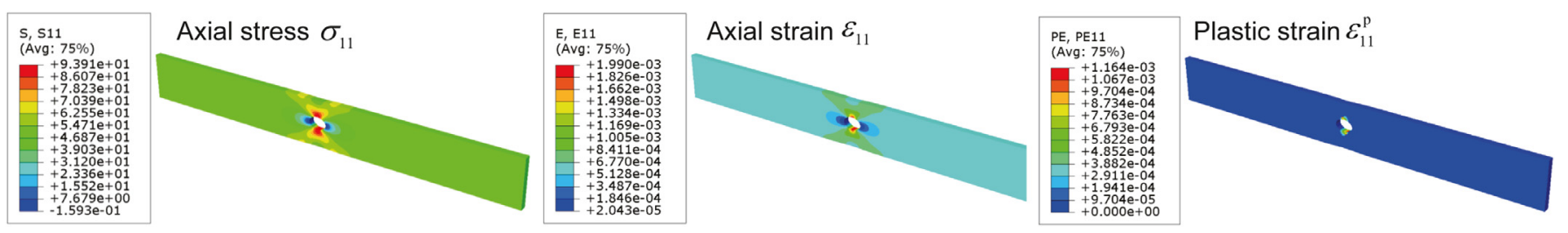

Fig. 13. The FEM results for the open-hole specimen under uniaxial tensile load.

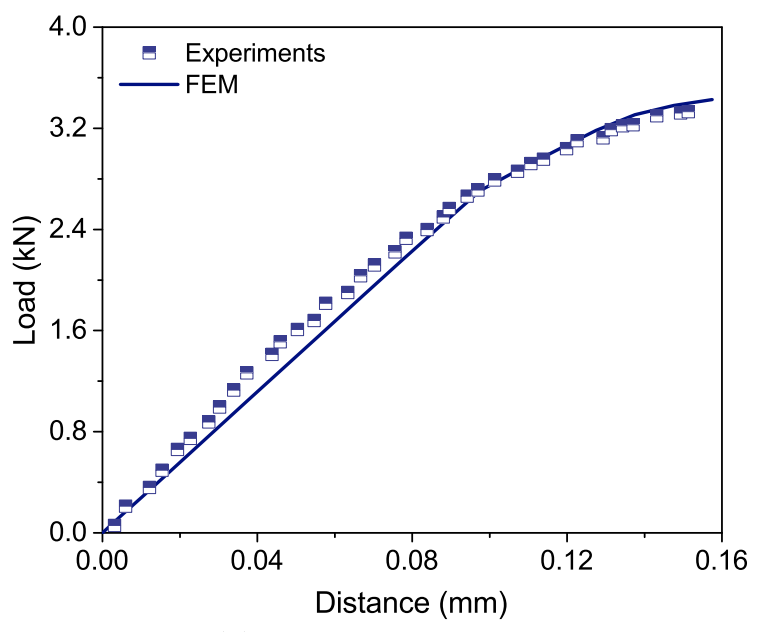

(a) Load-distance curves

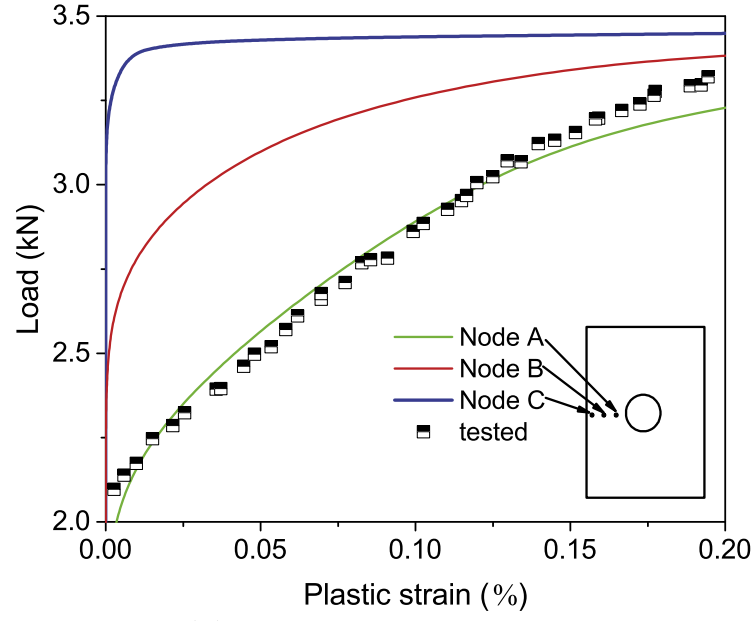

(b) Load-plastic strain curves

Fig. 14. Comparison of FEM calculation and experimental results.

\subsection{Finite element implementation}

The finite element implementation of the constitutive model is based on the discrete-time increments, and the backward Euler implicit integration method is used. Firstly, a trial stress tensor in step $n+1$ will be calculated as follows:

$\sigma_{n}^{\text {trail }}=\mathbf{S}: \Delta \varepsilon_{n}$

The parameter $A$ and $n$ can be determined by the fitting formula, then submit the trail equivalence stress into the yield surface function and calculate $f_{n}^{\text {trail }}$. If $f_{n}^{\text {trail }}<0$, the materials keep in the elastic region and there will be no additional plastic strains in this step, then elastic strain increment and plastic strain increment can be expressed as

$\left\{\begin{array}{l}\Delta \boldsymbol{\sigma}_{n}^{\mathrm{e}}=\mathbf{S}: \varepsilon_{n}^{\mathrm{e}} \\ \Delta \varepsilon_{n}^{\mathrm{e}}=\Delta \varepsilon_{n} \\ \Delta \varepsilon_{n}^{\mathrm{p}}=0\end{array}\right.$

Otherwise, the materials will come into yield and a plastic strain increment $\Delta \varepsilon_{n}^{\mathrm{p}}$ will appear. As the computational formula of stress increment, elastic strain increment and plastic strain increment is expressed as,

$\left\{\begin{array}{l}\Delta \sigma_{n}=\mathrm{S}: \varepsilon_{n}^{\mathrm{e}} \\ \Delta \sigma_{n}^{\mathrm{e}}=\Delta \varepsilon_{n}-\Delta \varepsilon_{n}^{\mathrm{p}} \\ \Delta \varepsilon_{n}^{\mathrm{p}}=\Delta \bar{\varepsilon}_{n}^{\mathrm{p}} \partial_{\sigma} f\end{array}\right.$

Submitting these expressions into the yield surface function, an equation with the independent variable $\Delta \bar{\varepsilon}_{n}^{p}$ can be determined as

$\bar{\sigma}\left(\sigma_{n}+\mathbf{S}:\left(\Delta \varepsilon_{n}-\Delta \bar{\varepsilon}_{n}^{\mathrm{p}} \partial_{\sigma} f\right)\right)-\kappa\left(\bar{\varepsilon}_{n}^{\mathrm{p}}+\Delta \bar{\varepsilon}_{n}^{\mathrm{p}}, \omega_{n}+\Delta \omega_{n}\right)=0$ which is solved by the Newton-Raphson iteration. The initial condition of the iteration procedure can be expressed as

$\Delta \varepsilon_{n}^{\mathrm{p}, 0}=0$

the following iterations are obtained,

$\Delta \varepsilon_{n}^{\mathrm{p}, k+1}=\Delta \varepsilon_{n}^{\mathrm{p}, k}-\frac{f\left(\Delta \varepsilon_{n}^{\mathrm{p}, k}\right)}{f^{\prime}\left(\Delta \varepsilon_{n}^{\mathrm{p}, k}\right)}$

until $f\left(\Delta \bar{\varepsilon}_{n}^{\mathrm{p}, k+1}\right) \leq \mathrm{TOL}$, where TOL $=1 \times 10^{-10}$ is the error tolerance in the present work, and the superscript $k$ is the number of iterations. Then $\varepsilon_{n}^{\mathrm{p}, k+1}, \bar{\varepsilon}_{n+1}^{\mathrm{p}, k+1}$ and $\sigma_{n}^{k+1}$ will be calculated and stored as updated solutiondependent variables (SDVs) after $\Delta \bar{\varepsilon}_{n}^{\mathrm{p}, k+1}$ is determined by the iteration procedure.

\subsection{Results}

Fig. 13 shows the distribution of $\sigma_{11}, \varepsilon_{11}, \varepsilon_{11}^{\mathrm{p}}$ in an open-hole specimen under tensile loading using the proposed model. The obvious stress concentration around the hole is observed, which is corresponding to the collapsed in the center-hole position in experiments. The location of the maximum stress is around the hole, perpendicular to the loading direction, where the plastic strain also exists.

Besides, the load-displacement curve calculated by FEM is compared with the experimental data in Fig. 14(a). The curve can be divided into two stages: the load grows linearly with the increase of the distance, and the elastic behavior is observed in the materials; and with the distance 
increases, the materials begin to be plastified, and the rate of increase for the load is decreased until the final failure. The computed curve shows a good agreement with the experiment data, and there is an acceptable error at the beginning of the curve, which is resulted from the damage existing in the matrix around the hole, and the fiber plays a major role in bearing loading, causing the modulus increases slightly.

Fig. 14 (b) demonstrates the evolution of load with increasing plastic strain. As the strain distribution is non-uniform around the hole, which is on account of the stress concentration effect proved by the stress distribution mentioned above, three points in the surface of the open-hole specimen model are chosen to accurately simulate the experimental strain measure point. The points are located in the centerline of the hole, with the distance of 1,2 , and $3 \mathrm{~mm}$ from the hole edge, as illustrated in Fig. 14(b). Then the evolutions of load versus plastic strain for the three nodes are calculated. The simulated plastic strain $\varepsilon_{11}^{\mathrm{p}}$ results of node-A coincide well with the experimental data, which means that the node-A location corresponding to the strain gage position. Besides, it also shows that under the same loading, the plastic strains of the points are grater when the points are closer to the hole, which has been validated by the plastic strain distribution. With the load increases, the plastic strain of the points away from the hole will generate rapidly and reach the critical value, leading to the final failure of the specimens.

Thus, it can be concluded that the proposed model can predict the mechanical behaviors of the material accurately via the comparison with the calculation results and experimental data of the open-hole specimen.

\section{Conclusion}

In the present work, an anisotropic elastic-plastic constitutive model is developed and verified for thermal shocked ox/ox-CMCs based on extensive tensile experimental results. The model accounts for the material response of the thermal shocked ox/ox-CMCs in the thermal shock temperatures, which can provide a sufficiently prediction of the stressstrain response for the thermal shocked ox/ox-CMCs under off-axis tensile loading. However, the complex effects of thermal aging and the number of thermal shocks are not considered in this model, which the ox/ox-CMCs often suffer form for the practical application, due to the limitation of experimental conditions. The following conclusions can be drawn:

- The thermal shocked ox/ox-CMCs displayed significant inelastic behavior related to plastic deformations as well as material degradation observed from on-axis and off-axis tensile experiments, which is related to the off-axis angles. The $0^{\circ} / 90^{\circ}$ specimens show much higher yield stress than the $45^{\circ}$ specimens. These characteristics indicate that the brittleness of the material in the fiber direction is obvious while the shear stress will induce the material to be ductile.

- The failure mechanism of the thermal shocked ox/ox-CMCs related to the loading conditions is revealed in the present analysis. For $0^{\circ}$ monotonic tensile specimen, the fracture surface is flat and the main feature of the fracture surface is cracks in matrix, yet for $45^{\circ}$ monotonic tensile specimen the fracture surface is irregular and the phenomenon of fiber bundle pull-out and single fiber pull-out can be observed.

- The constitutive model developed in the present work decomposes the off-axis and thermal shock temperature effects into plastic hardening. The plastic hardening is related to the off-axis tensile test, which provides the possibility to compare the hardening equation with experimental records directly.

- The variations of $\bar{\sigma}-\mathrm{d} \bar{\varepsilon}_{\mathrm{p}}$ relationships with the loading conditions were taken into account in the present model. Such formulation provides a simple correlation among the off-axis load and thermal shock effects and the material's principal direction.

- Introduction of the orientation-dependent and temperaturedependent hardening rule into the constitutive model provides a direct strategy to construct anisotropic plasticity for composites.
However, this strategy is perhaps only efficient for $2 \mathrm{D}$ materials or thin plates and needs further verifications based on more extensive experimental and computational investigations.

\section{Declaration of Competing Interest}

The authors declare that they have no known competing financial interests or personal relationships that could have appeared to influence the work reported in this paper.

\section{CRediT authorship contribution statement}

Zhengmao YANG: Conceptualization, Methodology, Software, Writing - review \& editing, Data curation, Writing - original draft, Supervision, Visualization. Hui LIU: Investigation, Validation.

\section{Acknowledgment}

The present work is supported by the Strategic Priority Research Program of Chinese Academy of Sciences (grant no. XDA17030100), the National Natural Science Foundation of China (grant no. 11572169 and 51775294).

\section{References}

[1] Genet M, Marcin L, Baranger E, Cluzel C, Ladevze P, Mouret A. Computational prediction of the lifetime of self-healing CMC structures. Compos Part A 2012;43(2):294-303.

[2] Longbiao L. In-phase thermomechanical fatigue damage evolution of long fiber-reinforced ceramic-matrix composites using fatigue hysteresis-based damage parameters. Int J Mech Sci 2018;140:189-99.

[3] Tushtev K, Almeida M, Saint R. 5.5 Oxide/Oxide CMCs -porous matrix composite systems; composites with interface coatings. Oxford: Elsevier; 2018, p. 130-57.

[4] Longbiao L. Damage evolution of fiber-reinforced ceramic-matrix composites under stress-rupture and cyclic loading at elevated temperature in oxidizing atmosphere. Int J Mech Sci 2017;131-132:938-55.

[5] Hui M, Laifei C, Litong Z, Yongdong X. Effect of fiber architectures on thermal cycling damage of $\mathrm{C} / \mathrm{SiC}$ composites in oxidizing atmosphere. Mater Sci Eng A 2007;460-461:306-13.

[6] Hay RS, Keller KA, Zawada LP, Jacobson NS, Fair GE. Degradation of Nextel ${ }^{\mathrm{TM}}$ 610-based oxide-oxide ceramic composites by aluminum oxychloride decomposition products. J Am Ceram Soc 2018;101(9):4203-23.

[7] Zhengmao Y, Junjie Y. Investigation of long-term thermal aged damage in oxide/oxide ceramic matrix composites. J Eur Ceram Soc 2019;40(4):1549-56.

[8] Jin ZH, Tohgo K, Fujii T, Shimamura Y, Noda N. Periodic surface cracks in an interpenetrating phase composite under a thermal shock. Int J Mech Sci 2018;149:583-90.

[9] Zhengmao Y, Huang Y, Markert B. Representation of micro-structural evolution and thermo-mechanical damage in thermal shocked oxide/oxide ceramic matrix composites. Int J Fatigue 2019;126:122-9.

[10] Rajan VP, Shaw JH, Rossol MN, Zok FW. An elasticcplastic constitutive model for ceramic composite laminates. Compos Part A 2014;66:44-57.

[11] Guiqiong J, Jun L, Bo W, Liang L, Chengpeng Y. Damage characteristics and constitutive modeling of the 2D C/SiC composite: part II - material model and numerical implementation. Chin J Aeronaut 2015;28(1):314-26.

[12] Budiansky B, Hutchinson JW, Evans AG. Matrix fracture in fiber-reinforced ceramics. J Mech Phys Solids 1986;34(2):167-89.

[13] Daxu Z, Hayhurst DR. Prediction of stress-strain and fracture behaviour of an 8-Harness satin weave ceramic matrix composite. Int $J$ Solids Struct 2014;51(21-22):3762-75.

[14] Chateau C, Gelebart L, Bornert M, Crepin J, Caldemaison D, Sauder C. Modeling of damage in unidirectional ceramic matrix composites and multi-scale experimental validation on third generation sic/sic minicomposites. J Mech Phys Solids 2014;63:298-319.

[15] Vinogradov V, Hashin Z. Probabilistic energy based model for prediction of transverse cracking in cross-ply laminates. Int J Solids Struct 2005;42(2):365-92.

[16] Chen JF, Morozov EV, Shankar K. A combined elastoplastic damage model for progressive failure analysis of composite materials and structures. Compos Struct 2012;94(12):3478-89.

[17] Rajan VP, Zok FW. Effects of non-uniform strains on tensile fracture of fiber-reinforced ceramic composites. J Mech Phys Solids 2012;60(12):2003-18.

[18] Santhosh U, Ahmad J, Ojard G, Miller R, Gowayed Y. Deformation and damage modeling of ceramic matrix composites under multiaxial stresses. Compos Part B 2016;90:97-106.

[19] Sun CT, Chen JL. A simple flow rule for characterizing nonlinear behavior of fiber composites. J Compos Mater 1989;23(10):1009-20.

[20] Vyas GM, Pinho ST, Robinson P. Constitutive modelling of fibre-reinforced composites with unidirectional plies using a plasticity-based approach. Compos Sci Technol 2011;71(8):1068-74. 
[21] Zarga D, Tounsi A, Bousahla AA, Bourada F, Mahmoud SR. Thermomechanical bending study for functionally graded sandwich plates using a simple quasi-3D shear deformation theory. Steel Compos Struct 2019;32(3):384-410.

[22] Karami B, Shahsavari D, Janghorban M, Tounsi A. Resonance behavior of functionally graded polymer composite nanoplates reinforced with graphene nanoplatelets. Int J Mech Sci 2019;156:94-105.

[23] Karami B, Janghorban M, Tounsi A. Wave propagation of functionally graded anisotropic nanoplates resting on winkler-pasternak foundation. Struct Eng Mech 2019;70(1):55-66.

[24] Chaabane LA, Bourada F, Sekkal M, Zerouati S, Zaoui FZ, Tounsi A, et al. Analytical study of bending and free vibration responses of functionally graded beams resting on elastic foundation. Struct Eng Mech 2019;71(2):185-96.

[25] Karami B, Janghorban M, Shahsavari D, Tounsi A. A size-dependent quasi-3D model for wave dispersion analysis of FG nanoplates. Steel Compos Struct 2018;28(1):99-110.

[26] Junbo X, Guodong F, Zhen C, Jun L. An anisotropic elastoplastic damage constitutive model for 3D needled C/C-SiC composites. Compos Struct 2017;176:164-77.

[27] Hui L, Zhengmao Y, Huang Y. A novel elastoplastic constitutive model for woven oxide/oxide ceramic matrix composites with anisotropic hardening. Compos Struct 2019;229:111420. doi:10.1016/j.compstruct.2019.111420.

[28] Zok FW, Levi CG. Mechanical properties of porous-matrix ceramic composites. Adv Eng Mater 2001;3(1-2):15-23.

[29] Ben Ramdane C, Julian-Jankowiak A, Valle R, Renollet Y, Parlier M, Martin E, et al. Microstructure and mechanical behaviour of a $\mathrm{Nextel}^{\mathrm{TM}}$ 610/alumina weak matrix composite subjected to tensile and compressive loadings. J Eur Ceram Soc 2017;37(8):2919-32.
[30] Zhengming H, Chunchun Z, Yuande X. Stiffness prediction of short fiber reinforced composites. Int J Mech Sci 2019;161-162:105068.

[31] Zhengmao Y, Hui L, Junjie Y. Damage constitutive model for thermal shockedceramic matrix composite. Chin J Theoret ApplMech 2019;51(6):1797-809. doi:10.6052/0459-1879-19-229.

[32] Zhengmao Y, Hui L. A continuum damage mechanics model for 2-D woven oxide/oxide ceramic matrix composites under cyclic thermal shocks. Ceram Int 2019;46(5):6029-37.

[33] Zhengmao Y, Hui L. Effects of thermal aging on the cyclic thermal shock behavior of oxide/oxide ceramic matrix composites. Mater Sci Eng A 2020;769:138494. doi:10.1016/j.msea.2019.138494.

[34] Ruggles-Wrenn MB, Lanser RL. Tension-compression fatigue of an oxide/oxide ceramic composite at elevated temperature. Mater Sci Eng A 2016;659:270-7.

[35] Zhengmao Y, Hui L, Huang Y. Micro-porosity as damage indicator for characterizing cyclic thermal shock-induced anisotropic damage in oxide/oxide ceramic matrix composites. Eng Fract Mech 2019;220:106669. doi:10.1016/ j.engfracmech.2019.106669.

[36] Zhengmao Y, Huang Y, Hui L. Evolution and characterization of cyclic thermal shock-induced thermomechanical damage in oxide/oxide ceramics matrix composites. Int J Fatigue 2019;120:150-61.

[37] Byrd LW, Birman V. Onset of matrix cracking in angle-ply ceramic matrix composites. Int J Mech Sci 2002;44(10):2173-87. 